\title{
Effects of Brief Adjunctive Metformin Therapy in Virologically Suppressed HIV-Infected Adults on Polyfunctional HIV-Specific CD8 T Cell Responses to PD-L1 Blockade
}

\author{
Glen M. Chew, ${ }^{1, \star}$ Ana Joy P. Padua, ${ }^{2, \star}{ }^{\star}$ Dominic C. Chow, ${ }^{1}$ Scott A. Souza, Danielle M. Clements, \\ Michael J. Corley, ${ }^{1, \dagger}$ Alina P.S. Pang, ${ }^{1}$ Marissa M. Alejandria, ${ }^{2}$ Mariana Gerschenson, ${ }^{1}$
} Cecilia M. Shikuma, and Lishomwa C. Ndhlovu, ${ }^{1, \dagger}$

\begin{abstract}
Targeting inhibitory immune checkpoint receptor pathways has shown remarkable success in improving anticancer T cell responses for the elimination of tumors. Such immunotherapeutic strategies are being pursued for HIV remission. Metformin has shown favorable clinical outcomes in enhancing the efficacy of programmed cell death-1 (PD-1) blockade and restoring antitumor T cell immunity. Furthermore, monocytes are known to be a strong predictor of progression-free survival in response to anti-PD-1 immunotherapy. In a single-arm clinical trial, we evaluated the immunological effects over an 8-week course of metformin therapy in seven euglycemic, virally suppressed HIV-infected participants on combination antiretroviral therapy (cART). We assessed changes in peripheral HIV-Gag-specific T cell responses to immune checkpoint blockade (ICB) with anti-PDL1 and anti-T cell immunoreceptor with immunoglobulin and ITIM domain (TIGIT) monoclonal antibodies (mAbs) and changes in CD8 T cell and monocyte subsets using flow cytometry. Study participants were all male, $71 \%$ (5/7) Caucasian, with a median age of 61 years, CD4 count of 739 cells/ $\mu \mathrm{L}$, and plasma HIV RNA of $<50$ copies/mL on stable cART for $>1$ year. Ex vivo polyfunctional HIV-Gag-specific CD8 T cell responses to anti-PD-L1 mAb significantly improved $(p<.05)$ over the 8-week course of metformin therapy. Moreover, frequencies of both intermediate $\left(\mathrm{CD} 14^{+} \mathrm{CD} 16^{+} ; r=0.89, p=.01\right)$ and nonclassical $\left(\mathrm{CD} 14^{\text {low }} \mathrm{CD} 16^{+} ; r=0.92\right.$, $p=.01$ ) monocytes at entry were predictive of the magnitude of the anti-HIV CD8 T cell responses to PD-L1 blockade. Collectively, these findings highlight that 8-week course of metformin increases the polyfunctionality of CD8 T cells and that baseline monocyte subset frequencies may be a potential determinant of PD-L1 blockade efficacy. These data provide valuable information for HIV remission trials that utilize ICB strategies to enhance anti-HIV CD8 T cell immunity.
\end{abstract}

Keywords: HIV, metformin, T cells, PD-1, PD-L1, immunotherapy

\section{Introduction}

D ESPITE BEING SUCCESSFUL in controlling viral replication, combination antiretroviral therapy (cART) alone does not eradicate HIV and people living with HIV have to adhere to lifelong therapy. There are considerable ongoing efforts to eliminate the HIV reservoir or reduce latent HIV-infected cells and allow for natural immune control in the absence of cART.

Expansion of key negative immune checkpoint receptors (NCRs), such as programmed cell death-1 (PD-1) and T cell immunoreceptor with immunoglobulin and ITIM domains (TIGIT), have been associated with $\mathrm{T}$ cell immune exhaustion, dysfunctional $\mathrm{T}$ cell effector responses and HIV reservoir size. ${ }^{1-3}$ In oncology, targeting NCR pathways has shown promise in reversing $\mathrm{T}$ cell exhaustion and improving long-term progression-free survival in a subset of cancer patients. $^{4}$

In the setting of HIV, blockade of the PD-1/PD-L1 pathway with either ipilimumab or nivolumab in HIV-infected cancer patients receiving cART resulted in decreased plasma viral load and a reduction in cell-associated HIV DNA. ${ }^{5,6}$ In a

\footnotetext{
${ }^{1}$ John A. Burns School of Medicine, University of Hawai'i, Honolulu, Hawaii, USA

${ }^{2}$ College of Medicine, University of the Philippines, Manila, Philippines.

*Both these authors contributed equally to this work and are considered to be co-first authors.

${ }^{\dagger}$ Current affiliation: Division of Infectious Diseases, Department of Medicine, Weill Cornell Medicine, New York, New York, USA.
} 
separate study, a single dose of an anti-PD-L1 monoclonal antibody $(\mathrm{mAb})$ increased HIV-specific CD8 $\mathrm{T}$ responses ${ }^{7}$ suggesting that NCR blockade may drive HIV reactivation, exposing infected cells to immune-mediated killing ${ }^{8}$ while reversing CD8 $\mathrm{T}$ cell immune exhaustion. Additional immune parameters, such as classical monocyte frequency, were shown to be a predictive biomarker for metastatic melanoma patients who responded to anti-PD-1 immunotherapy.

Understanding the mechanisms that would enhance the efficacy of immune checkpoint blockade (ICB) and determine predictors of response to immunotherapy would permit broad responses across participants in the setting of HIV remission and curative interventions.

Metformin is a safe and widely administered oral hypoglycemic biguanide used as a first-line therapy to treat type II diabetes mellitus, and it is now known to have beneficial pleiotropic immune effects resulting in anti-inflammatory and antineoplastic properties, which are independent of its glucoselowering ability. ${ }^{10}$ Metformin therapy results in the inhibition of the mitochondrial respiratory-chain complex-1 inhibition through AMP-activated protein kinase (AMPK)-dependent and independent downstream effects ${ }^{11}$ regulating cellular functions, including proliferation, inflammation, and immune responses such as the formation of memory CD8 T cells. ${ }^{12}$

Previous studies have reported the additive effects of metformin on improving ICB efficacy in the setting of cancer. Metformin potentiated the effects of PD-1 blockade on antitumor $\mathrm{T}$ cells by remodeling the hypoxic tumor microenvironment. ${ }^{13}$ Moreover, metformin combined with anti-PD-1 and anti-CTLA-4 was shown to have favorable treatmentrelated outcomes in metastatic malignant melanoma patients. ${ }^{14}$ Based on these observations, the pleiotropic effects of metformin may also improve $\mathrm{T}$ cell immunity in response to ICB in other chronic diseases, such as HIV infection. In this study, we provided insight on the possible impact of a short course of adjunctive metformin therapy in HIV-infected individuals on suppressive cART by measuring changes in peripheral anti-HIV T cell responses to ICB.

\section{Materials and Methods}

\section{Ethics statement}

All persons gave written informed consent to participate in the study and IRB approval for the study was obtained from the University of Hawaii Human Studies Program. In addition, all participants signed a separate informed consent document agreeing to the use of their banked specimens for other research related to HIV.

\section{Study participants and study schema}

This was an open label, 8-week pilot study in seven euglycemic adults on cART, stable for $>1$ year with last plasma HIV RNA $<50$ copies/mL measured within the last 6 months of enrollment. Exclusion criteria included uncontrolled chronic medical conditions or cancers, acute illness within 2 weeks of entry, diagnosis of diabetes, history of chronic diarrhea, untreated hepatitis $\mathrm{C}$ coinfection, and laboratory parameters of hemoglobin $<9.0 \mathrm{~g} / \mathrm{dL}$, absolute neutrophil count $<1,000 / \mathrm{mL}$, platelet count $<50,000 / \mathrm{mL}$, AST and ALT
$>5 \times$ upper limit of normal (ULN), and creatinine clearance by Cockcroft and Gault of $<60 \mathrm{~mL} / \mathrm{min}$. Adjunctive metformin extended-release dosing was $500 \mathrm{mg}$ at entry to week 3 , increasing to $1,000 \mathrm{mg}$ at week 4 until end of study at week 8 .

\section{Immunophenotyping}

Cryopreserved peripheral blood mononuclear cells (PBMCs) were thawed in complete RPMI 1640 (cRPMI; HyClone) containing $10 \%$ fetal bovine serum (FBS) (Hyclone), 1\% penicillin/streptomycin (HyClone), $10 \mathrm{mM}$ HEPES (HyClone), $2 \mathrm{mM}$ L-glutamine (Hyclone), and washed with $1 \times$ phosphate-buffered saline (PBS; HyClone) before staining with Live/Dead aqua amine reactive dye (AARD; Invitrogen) or yellow amine reactive dye (YARD; Invitrogen). Cells were washed again with PBS supplemented with $2 \%$ FBS followed by panels of fluorochrome-conjugated antihuman mAbs to identify lymphocyte populations expressing NCRs, markers of activation, senescence and proliferation, or monocyte compartments: Beckman Coulter: ECD-conjugated anti-CD3 (Clone; UCHT1) mAb. BD Biosciences: Alexa Fluor 700-conjugated anti-CD4 (RPA-T4), V450-conjugated antiCD45RA (HI100), PE-Cy7-conjugated anti-CD19 (SJ23C1), PE-Cy7-conjugated anti-CD20 (2H7), PE-CF549-conjugated anti-CD7 (M-T701), FITC-conjugated anti-CD57 (NK-1), and PE-Cy5-conjugated anti-CD38 (HIT2) mAbs. BioLegend: Alexa Fluor 647-conjugated anti-CCR7 (G043H7), APC-Cy7-conjugated anti-PD-1 (EH12.2H7), Brilliant Violet (BV) 605-conjugated anti-CD14 (M5E2), BV 711-conjugated anti-CD16 (3G8), APC-Cy7-conjugated anti-HLADR (L243), BV 510-conjugated anti-CD11b (ICRF44), BV 711-conjugated anti-CD3 (OKT3), and Alexa Fluor 674-conjugated Ki-67 [intracellular stain (L428)]. eBioscience: PE-Cy7-conjugated antiCD28 (CD28.2), PerCP-eFluor 710-conjugated anti-TIGIT (MBSA43). R\&D Systems: PE-conjugated anti-TIM-3 (344823) mAbs. Thermo Fisher Scientific: Qdot605-conjugated anti-CD8 (3B5) mAb. Cells were washed and fixed with $1 \%$ paraformaldehyde before acquiring on a custom BD Biosciences four-laser LSR Fortessa flow cytometer in our laboratory. Isotype controls or fluorescence minus one (FMO) samples were used to facilitate gating. UltraComp eBeads (Thermo Fisher Scientific) were individually stained with fluorochrome-conjugated antibody and used for softwarebased compensation. Data were analyzed using FlowJo software version 10.3 (Treestar).

\section{Peptide stimulation}

One hundred and twenty-three overlapping $\sim 15$ mer HIV1 clade B gag peptides obtained from the National Institutes of Health (NIH) AIDS Reagent Program. Stimulations were performed with a final concentration of $10 \mu \mathrm{g} / \mathrm{mL}$ of peptide. Anti-CD3+anti-CD28 mAb Dynabeads (Life Technologies) were used as positive control stimulation following the manufacturer's recommended procedure.

\section{Anti-TIGIT and anti-PD-L1 mAbs}

The anti-TIGIT mAb (clone 11G11) and anti-PD-L1 mAb (clone 12A4) were a generous gift from BMS and have been previously described. ${ }^{1}$ 
Table 1. Study Participant Characteristics

\begin{tabular}{ll}
\hline Characteristics & Metformin $(\mathrm{n}=7)$ \\
\hline Age, years & $61(50$ to 65$)$ \\
Gender, male & $7(100)$ \\
Ethnicity, Caucasian & $5(71)$ \\
Fasting glucose, $\mathrm{mg} / \mathrm{mL}$ & $107(99$ to 112$)$ \\
Current CD4 counts, cell $/ \mu \mathrm{L}$ & $739(659$ to 1,083$)$ \\
Plasma HIV RNA, copies/mL & Undetectable $(<50)$ \\
\hline
\end{tabular}

Median (Q1 to Q3) presented for continuous variables, $n(\%)$ presented for categorical variables.

\section{Ex vivo functional blockade assay}

PBMCs were rapidly thawed in cRPMI and seeded at one million cells per condition. Cell cultures were then stimulated with pooled HIV Gag peptides in the presence or absence of blocking mAb targeting PD-L1 or TIGIT. Briefly, on day 0, cell cultures were stimulated with HIV peptide pool+PD-L1 and/or TIGIT blocking mAbs. On day 2, interleukin (IL)-2 (50 IU/mL) was added to induce $\mathrm{T}$ cell expansion. On day 5, cells were washed with cRPMI and restimulated with original conditions in addition to $5 \mu \mathrm{g} / \mathrm{mL}$ of brefeldin A and $5 \mu \mathrm{g} / \mathrm{mL}$ of monensin (Sigma-Aldrich) and APC-conjugated antiCD107a (H4A3). Cells were harvested on day 6 for immunophenotyping and intracellular cytokine staining. Cells were stained with Live/Dead AARD followed by fluorochromeconjugated mAbs against CD4 (RPA-T4; APC-Cy7) and CD8 (3B5; Qdot605) followed by intracellular staining with ECD-conjugated anti-CD3 (UCHT1), FITC-conjugated anti-interferon gamma (IFN- $\gamma$ ), Alexa700-conjugated antitumor necrosis factor-alpha (TNF- $\alpha$; MAb11), and BV421conjugated anti-IL-2 (MQ1-17H12). Cells were acquired and analyzed as described above.

\section{Monocyte intracellular cytokine staining assay}

Cryopreserved PBMC were thawed in serum-free media (AIM V Medium; Life Technologies) containing $10 \mu \mathrm{g} / \mathrm{mL}$ of DNAse (Sigma) rested overnight at $37^{\circ} \mathrm{C}$ and $5 \% \mathrm{CO}_{2}$ in a polypropylene $96-w e l l$ plate. The next day, the cells were stimulated with either $100 \mathrm{ng} / \mathrm{mL}$ lipopolysaccharide (LPS; Invitrogen) or media alone (unstimulated) for $6 \mathrm{~h}$ in the presence of brefeldin-A $(5 \mu \mathrm{g} / \mathrm{mL})$ and monensin $(5 \mu \mathrm{g} / \mathrm{mL})$. After surface staining with BV711-conjugated anti-CD3, BV605conjugated anti-CD14, BV421-conjugated anti-CD16, PECy7-conjugated anti-CD7, PE-Cy7-conjugated, anti-CD19, PE-Cy7-conjugated anti-CD20, and PerCP-Cy5.5-CD11bconjugated anti-CD11b, APC-H7-conjugated anti-HLA-DR $\mathrm{mAb}$, and with Live/Dead fixable YARD, cells were fixed, permeabilized (BD FACS Lyse, Perm Buffer II), and intracellularly stained with $\mathrm{mAb}$ obtained from BioLegend: APC-conjugated anti-IL-6 (MQ2-13A5), FITC-conjugated anti- IL-8 (BH0814), and eBioscience: PE-conjugated anti-IL-
$1 \beta$ (AS10) and Alexa Fluor700-conjugated anti-TNF- $\alpha$ (MAb11). Cells were acquired and analyzed.

\section{Statistical analysis}

Nonparametric Wilcoxon matched-pair signed rank test was used to compare changes between entry weeks 0 and 8 in CD8 T cell phenotype and function. Spearman's rho was used for correlations. A $p$ value $<.05$ was regarded as statistically significant for all analyses and all results were conducted using SAS v9.4.

\section{Results}

Frequency of central memory CD8 T cells in peripheral blood increases following brief 8-week intensification with $1,000 \mathrm{mg}$ metformin

The study participants consisted of seven euglycemic virally suppressed HIV-infected individuals with a median age of 61 years old, all male, with a median CD4 count of 739 cells/ $\mu \mathrm{L}$ (Table 1 ). The short course of adjunctive metformin was taken equally and was well tolerated up to $1,000 \mathrm{mg} /$ day during the entire study period by all participants. CD8 T cell memory populations were assessed by flow cytometry (Fig. 1A). We observed a significant increase in central memory $\left(\mathrm{CD} 45 \mathrm{RA}^{-} \mathrm{CCR}^{+} \mathrm{CD} 28^{+}\right.$) from entry week 0 [median $8.1 \%$ (interquartile range, 5.3 to 11.7$)]$ vs. week 8 [12.2\% (6.03 to $13.8 ; p=.03$ )] (Fig. 1B, C), whereas no significant changes were observed in naive $\left(\mathrm{CD} 45 \mathrm{RA}^{+} \mathrm{CCR} 7^{+} \mathrm{CD} 28^{+}\right)$, transitional memory $\left(\mathrm{CD} 45 \mathrm{RA}^{-} \mathrm{CCR} 7^{+} \mathrm{CD} 28^{+}\right)$, effector memory (CD45RA ${ }^{-} \mathrm{CCR}^{-} \mathrm{CD}^{-} 8^{-}$), or terminally differentiated (CD45 $\left.\mathrm{RA}^{+} \mathrm{CCR} 7^{-} \mathrm{CD} 28^{-}\right) \mathrm{CD} 8 \mathrm{~T}$ cell subsets. Furthermore, we did not observe significant increases in $\mathrm{CD} 8 \mathrm{~T}$ cell activation $\left(\mathrm{CD}_{3}{ }^{+} \mathrm{HLA}^{-} \mathrm{DR}^{+}\right)$, senescence $\left(\mathrm{CD} 57^{+} \mathrm{CD} 28^{-}\right)$, or proliferation measured by intracellular $\mathrm{Ki}-67$ staining comparing entry to week 8 of adjunctive metformin therapy (data not shown).

\section{Metformin therapy results in increased dual NCR-expressing PD-1 $1^{+}$TIGIT $T^{+}$CD8 T cells}

Since PD-1 and TIGIT immune checkpoint receptors have been shown to negatively regulate HIV-specific CD8 T cell responses, ${ }^{1,15,16}$ we next examined the frequencies of PD-1 and TIGIT expression on CD8 T cells in the blood. We found a modest increased frequency of PD- $1^{+} \mathrm{CD} 8 \mathrm{~T}$ cells after a short course of metformin therapy week $0[14.7 \%$ (8.83 to $17.8)]$ vs. week 8 [15.4\% (13.5 to 19$)](p=.07)$ and TIGIT $^{+}$ CD8 T cells week 0 [40.7\% (35.4 to 48.2$)]$ vs. week 8 [41.9 (37 to 62.7)] ( $p=.07$ ) (data not shown). We, however, observed a significant increase in dual NCR-expressing PD$1^{+}$TIGIT $^{+}$CD8 $\mathrm{T}$ cells after 8 weeks of metformin therapy compared with baseline week $0[10.9 \%$ (5.8 to 12.1$)]$ vs. week 8 [11.9\% (8.4 to 14.4)] $(p=.03)$ (Fig. 1D, E).

FIG. 1. Impact of 8 -week course of adjunctive metformin on frequencies of CD8 T cell subsets, and PD- $1^{+}$and TIGIT ${ }^{+}$ CD8 $\mathrm{T}$ cells. Representative flow cytometry plots of (A) CD8 $\mathrm{T}$ cell subsets, (B) the CM CD8 T cell subset $\left(\mathrm{CD} 45 \mathrm{RA}^{-} \mathrm{CCR}^{+} \mathrm{CD} 28^{+}\right.$) and $(\mathbf{C})$ changes in frequency of $\mathrm{CM} \mathrm{CD} 8 \mathrm{~T}$ cells. Representative flow cytometry plots of (D) PD1 and TIGIT coexpression on total CD8 $\mathrm{T}$ cells and (E) changes in frequency of TIGIT ${ }^{+} \mathrm{PD} 1^{+} \mathrm{CD}^{-} \mathrm{T}$ cell $(n=7)$. CM, central memory; PD-1, programmed cell death-1; TIGIT, T cell immunoreceptor with immunoglobulin and ITIM domains. Color images are available online. 

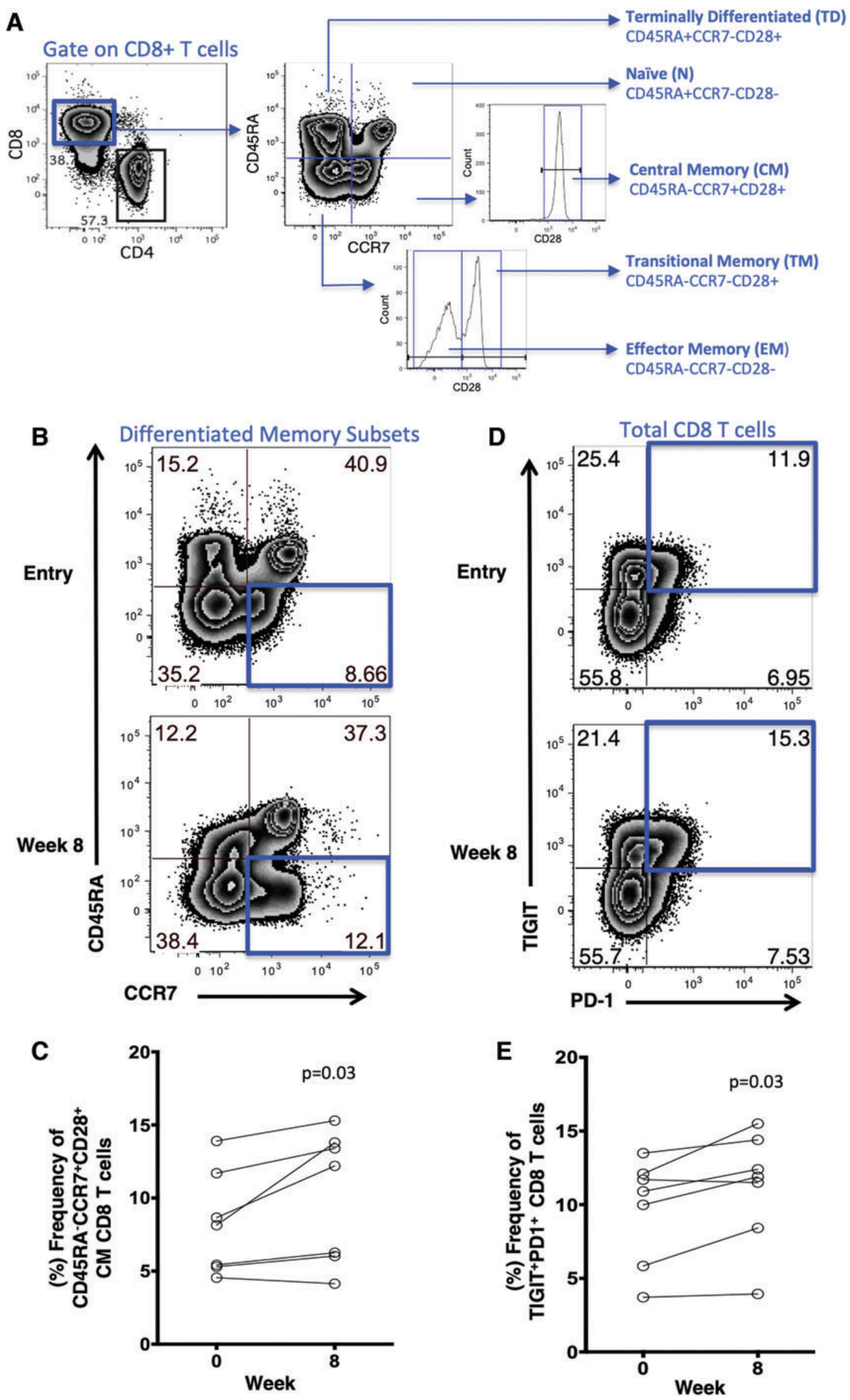
Monocyte populations and functional responses not impacted by brief metformin therapy

Metformin has been shown to impact monocyte responses in vitro. ${ }^{17} \mathrm{We}$ assessed the frequency of various monocyte subsets and observed no change in the frequency $(\%)$ of classical monocytes $\left(\mathrm{CD} 14^{+} \mathrm{CD}^{-} 6^{-}\right)$week 0 [91.6\% (89.5 to $97.1)]$ vs. week 8 [91\% (89.3 to 96.6)] $(p=.68)$, intermediate (inflammatory) monocytes $\left(\mathrm{CD} 14^{+} \mathrm{CD} 16^{+}\right)$week $0[4.28 \%$ (1.27 to 6.05)] vs. week 8 [5.14\% (1.66 to 8.11)] $(p=.15)$, or nonclassical (patrolling) monocytes $\left(\mathrm{CD} 14^{\mathrm{dim}} \mathrm{CD} 16^{+}\right)$week 0 [1.1\% (0.71 to 4.26$)]$ vs. week 8 [2.33\% (0.57 to 5.07$)]$ $(p=.94)$ subsets. We also assessed polyfunctional monocyte IL- $1 \beta$, IL- 6 , IL- 8 , and TNF- $\alpha$ responses to LPS through flow cytometry (Supplementary Fig. S1A) and observed no changes over the 8-week period in these inflammatory cytokines (Supplementary Fig. S1B). These data reveal the maintenance and stability of the peripheral monocyte population during a brief course of adjunctive metformin therapy.

\section{Metformin therapy alone does not impact ex vivo HIV-Gag-specific CD4 and CD8 T cell responses}

We next assessed the impact of adjunctive metformin on polyfunctional anti-HIV CD4 and CD8 T cell responses. We stimulated PBMCs with HIV Gag peptides and measured the HIV-Gag-specific degranulation (CD107a), IFN- $\gamma$, TNF- $\alpha$, and IL-2 effector responses at entry weeks 0 and 8 . We did not observe significant changes in mono or polyfunctional CD4 and CD8 $\mathrm{T}$ cell responses from entry weeks 0 to 8 (Supplementary Figs. S2-S4). There was no association seen between mono or polyfunctional HIV-Gag-specific CD4 and CD8 $\mathrm{T}$ cell responses and monocyte populations at weeks 0 and 8 (data not shown). These data suggest that metformin minimally impacts HIV-Gag-specific CD4 and CD8 T cell responses.

\section{Ex vivo HIV-Gag-specific polyfunctional responses to PD-L1 blockade improved following metformin therapy}

Given the increase in PD- $1^{+}$and TIGIT $^{+}$CD8 T cells over the course of adjunctive metformin, we next assessed the ability of ICB to improve anti-HIV CD8 T cell responses ex vivo. We stimulated $\mathrm{PBMCs}$ with HIV Gag peptides in the presence of clinical-grade mAbs blocking the PD-1 and TIGIT pathways. We observed a significant increase in HIVGag-specific CD8 T cell degranulation (CD107a expression) with anti-PD-L1 mAb after 8 weeks of metformin therapy compared with entry week 0 [fold change of isotype to antiPD-L1 mAb at week $0[-0.23 \%(-0.23$ to 0.09$)]$ vs. week 8 [ $-0.11(-0.26$ to 0.44$)](p=.04)$ (Fig. 2A, B). Furthermore, we observed a significant increase in polyfunctional HIVGag-specific CD8 T cells with anti-PD-L1 mAb after 8 weeks of metformin therapy compared with entry week 0 in CD107 ${ }^{+}$IFN $-\gamma^{+}$TNF- $\alpha^{+}$CD8 $\mathrm{T}$ cell fold change week 0 [ $-0.0485(-0.12$ to 0.18$)]$ vs. week 8 [0.23 (0.04 to 0.76$)]$ $(p=.04), \mathrm{CD} 107 \mathrm{a}^{+} \mathrm{IFN}-\gamma^{+} \mathrm{IL}-2^{+} \mathrm{CD} 8 \mathrm{~T}$ cell fold change week 0 [0.19 (-0.28 to 0.42$)]$ vs. week $8[1.21(-0.07$ to 2.40$)]$ $(p=.04)$, and CD107a ${ }^{+} \mathrm{IFN}-\gamma^{+} \mathrm{TNF}-\alpha^{+} \mathrm{IL}-2^{+} \mathrm{CD} 8 \mathrm{~T}$ cell fold change week $0[-0.11(-0.39$ to 0.53$)]$ vs. week $8[0.93(0.25$ to 2.72$)](p=.03)$ (Fig. 2C-E). No significant differences were seen with anti-TIGIT mAb at week 0 vs. week 8 (data not shown). Dual blocking with anti-PD-L1 mAb and antiTIGIT mAb compared with single blocking with anti-TIGIT $\mathrm{mAb}$ at week 0 vs. week 8 also did not result in significant differences (data not shown). Collectively, these findings suggest that PD-L1 blockade alone and not dual blockade could have an additive effect with metformin in improving the HIV-Gag-specific polyfunctional responses.

\section{Monocyte subsets and multiple NCR-expressing \\ CD8 T cells correlate with the magnitude of HIV-Gag-specific polyfunctional responses to ex vivo $P D$-L1 blockade after an 8-week course of metformin therapy}

Monocyte frequencies have been identified as potential biomarkers of cancer individuals who would likely respond to immunotherapy. ${ }^{9}$ Therefore, we next evaluated the frequency of monocyte subsets at baseline in relation to ex vivo HIV-Gag-specific CD8 T cell ICB and T cell-exhausted phenotypes. The entry week 0 frequency $(\%)$ of intermediate (inflammatory) monocytes $\left(\mathrm{CD} 14^{+} \mathrm{CD} 16^{+} ; r=0.89, p=.01\right)$ and nonclassical (patrolling) monocytes (CD14 ${ }^{\mathrm{dim}} \mathrm{CD} 16^{+}$; $r=0.92, p=.01$ ) correlated positively with the 8-week fold change of HIV-Gag-specific polyfunctional (CD107a ${ }^{+}$IFN$\gamma^{+} \mathrm{IL}_{-}{ }^{+}$) $\mathrm{T}$ cell response to PD-L1 blockade. On the other hand, classical monocytes (CD14 $\left.{ }^{+} \mathrm{CD}^{-} 6^{-} ; r=-0.78, p=.04\right)$ correlated negatively with CD8 $\mathrm{T}$ cell polyfunctionality (Fig. 3A-C). We also looked at the association of HIV-Gagspecific polyfunctional T cell responses to PD-L1 blockade and single or multiple negative checkpoint receptors (PD-1, TIGIT, TIM-3, and LAG-3) on CD4 and CD8 T cells at entry week 0 . Single- and dual NCR-expressing CD8 T cells as well as NCR-expressing CD4 T cells did not show correlation with polyfunctional T cell responses after PD-L1 blockade (data not shown). The entry week 0 frequencies of PD- $1^{+}$TIGIT $^{+} \mathrm{TIM}^{+}$ $(r=-0.78, p=.04)$ and PD- $1^{+}$TIGIT $^{+}$TIM $^{+}{ }^{+}$AAG $^{+}{ }^{+}(r=-0.82$, $p=.03) \mathrm{CD} 8 \mathrm{~T}$ cells correlated negatively with HV-Gag-specific

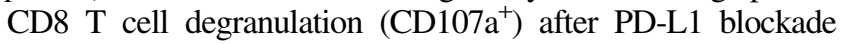
(Fig. 3D, E). These data suggest that the combination of the baseline monocyte subsets and degree of CD8 T cell exhaustion could function as predictors of the possible combinatorial effect of metformin and PD-L1 blockade on HIV-specific CD8 T cell responses.

\section{Discussion}

While cART has drastically improved the disease progression of HIV infection, it has since then been associated with chronic non-AIDS comorbidities. ${ }^{18}$ Treatment strategies that reduce inflammation and improve immune function to better health and target the viral reservoirs are currently being sought after. In this study, we demonstrate that an 8 -week short course of adjunctive metformin in nondiabetic virally suppressed HIV-infected individuals increases central memory CD8 T cells. Furthermore, metformin therapy improves PD-L1 blockade-enhanced HIV-specific CD8 T cell responses while associating with baseline frequencies of monocyte and NCR-expressing CD8 T cells. Taken together, adjunctive metformin may have an additive effect in improving ICB by enhancing HIV-specific CD8 T cell function, which is important to eliminate virally infected cells in HIV cure strategies. 
A
Entry

Week 8

Week 8

Week 8

Entry

Entry

Week 8

Entry

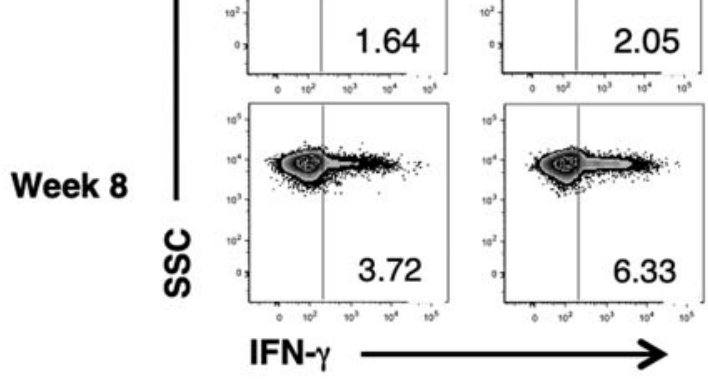

IL-2
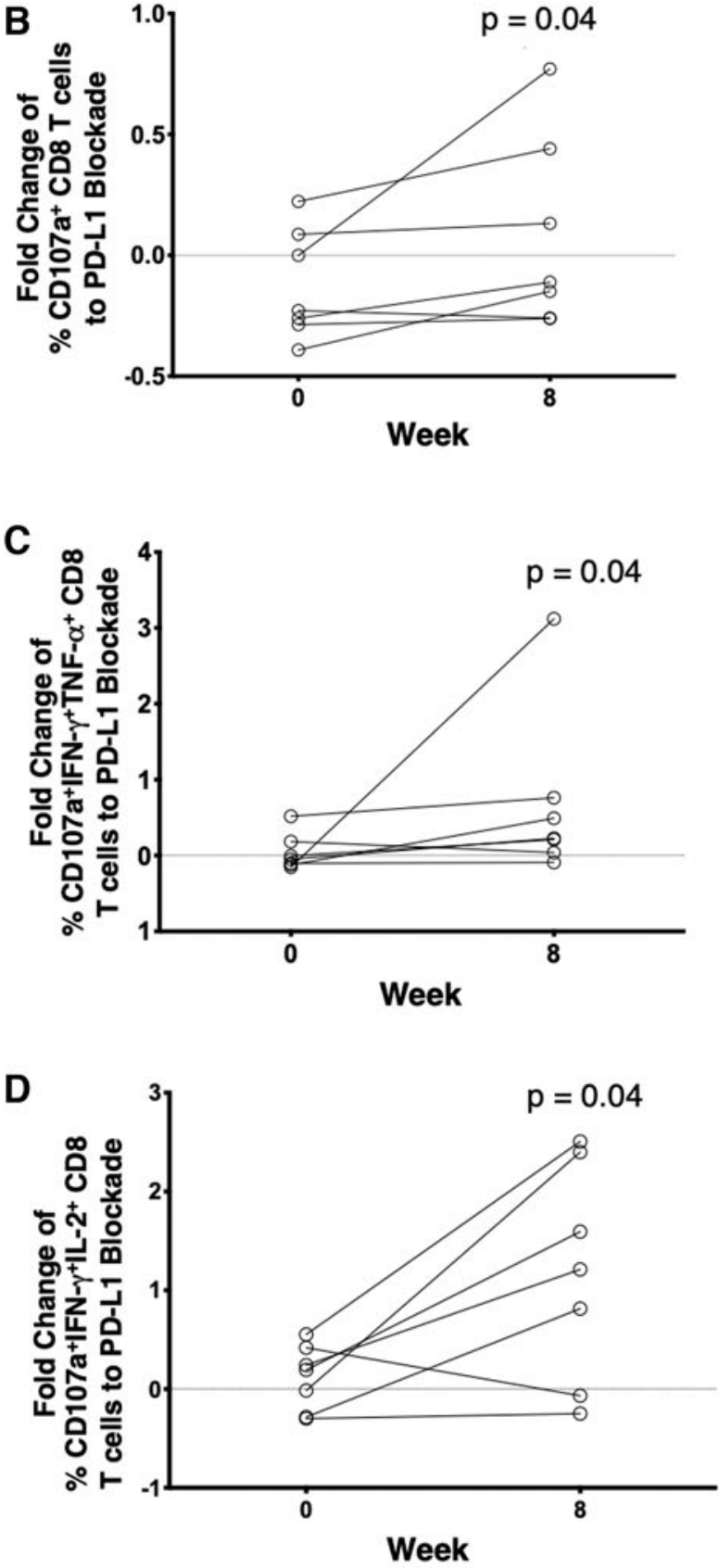

E

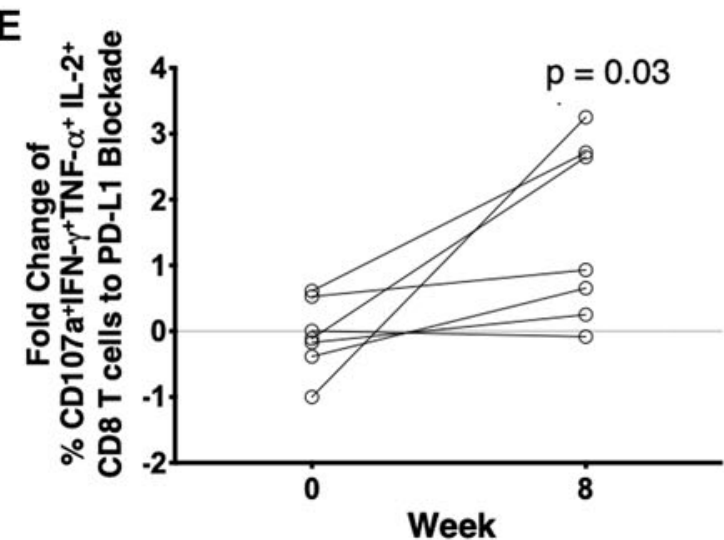

FIG. 2. Anti-PD-L1 blockade enhancement of adjunctive metformin on HIV-specific CD8 T cells ex vivo. (A) Representative flow cytometry plots showing CD107a, TNF- $\alpha$, IL-2, and IFN- $\gamma$-producing HIV-specific CD8 T cells at entry and week 8 of adjunctive metformin therapy with PD-L1 blockade or isotype. Fold changes of isotype to PD-L1 blockade in the frequencies of anti-HIV, (B) CD8 T cell degranulation (CD107a expression), and (C-E) polyfunctional CD8 T cell responses before and after metformin therapy $(n=7)$. IFN- $\gamma$, interferon gamma; IL, interleukin; TNF- $\alpha$, tumor necrosis factor-alpha. 

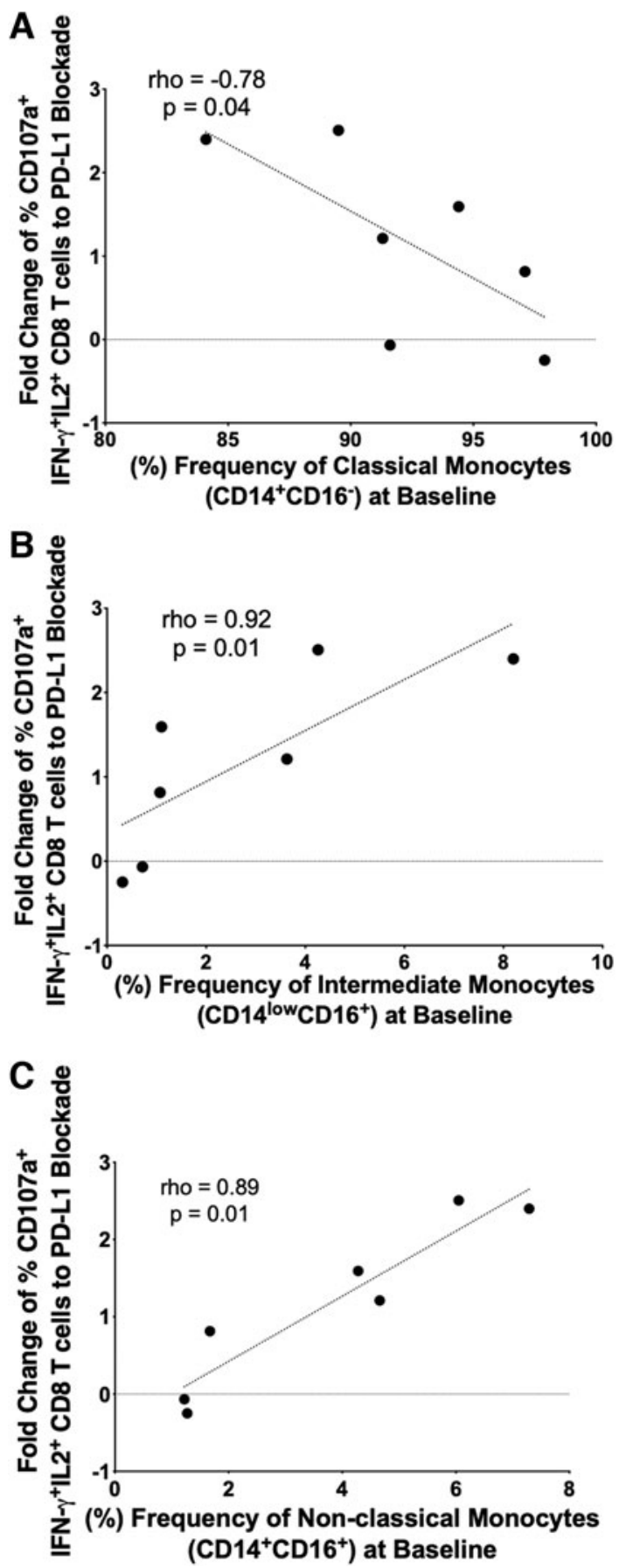

D

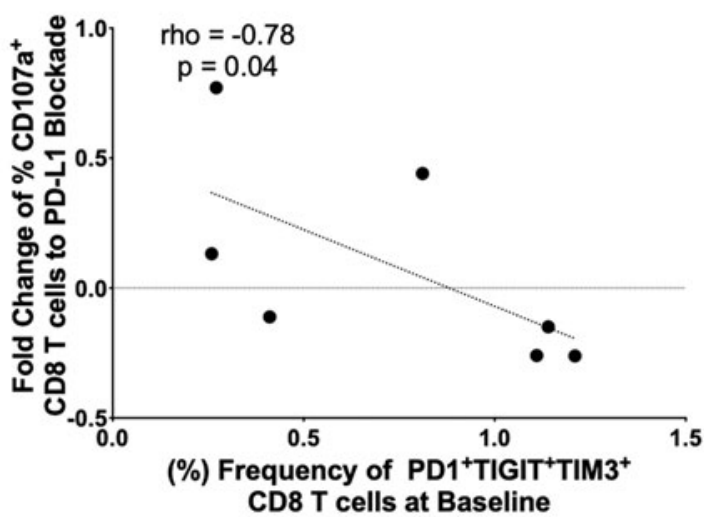

E

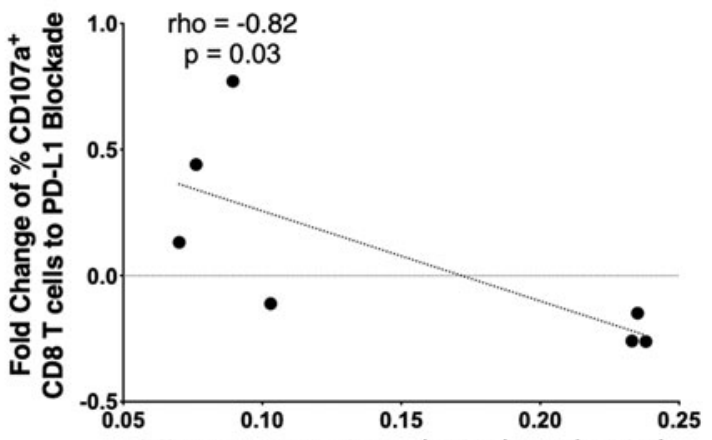

(\%) Frequency of $\mathrm{PD1}^{+} \mathrm{TIGIT}^{+} \mathrm{TIM}^{+} \mathrm{LAG3}^{+}$ CD8 $\mathrm{T}$ cells at Baseline

FIG. 3. NCR-expressing CD8 $\mathrm{T}$ cells and monocyte frequencies and anti-PD- L1-enhanced CD8 T cell polyfunctional correlations. Entry week 0 (baseline) frequency of (A) classical monocytes, (B) intermediate monocytes, (C) nonclassical monocytes correlate with the frequency fold change of polyfunctional (CD107a ${ }^{+}$IFN $-\gamma^{+}$IL- $2^{+}$) anti-HIV CD8 T cells, (D) triple-expressing NCR CD8 T cells, and (E) quadruple-expressing NCR CD8 T cells correlate with the frequency fold change of CD107a ${ }^{+}$anti-HIV CD8 T cells at week 8 of adjunctive metformin therapy with PD-L1 blockade ex vivo ( $\left.n=7\right)$. NCR, negative immune checkpoint receptor.

Acquired cell-mediated immunity is critical for the control and clearance of many human disease-causing infections. ${ }^{19}$ In HIV, CD8 T cells are important for maintaining viral suppression in the presence of Cart. ${ }^{20,21} \mathrm{We}$ observed a significant increase in the central memory CD8 $\mathrm{T}$ cells suggesting that metformin may be expanding a subset of pre-existing antigen- experienced central memory CD8 $\mathrm{T}$ cells. This may include pre-existing potent HIV-specific immune cells that recognize different variant HIV epitopes that may differentiate into effector cells to elicit protective immunity. ${ }^{22}$ Moreover, central memory CD8 T cells can also traffic into tissues and can become tissue resident cells and provide improved tissue 
surveillance in shock and kill HIV remission strategies. ${ }^{23}$ Our observations of increased central memory population CD8 T cells may have a beneficial effect on improving HIV-specific CD8 $\mathrm{T}$ cell responses.

The duration of metformin therapy may impact immunological outcomes. We have recently shown that a long course of metformin over 24 weeks led to decreases in PD- $1^{+}$TIGIT $^{+}$ and PD- $1^{+}$TIGIT $^{+}$TIM- $3^{+}$CD4 T cells, with limited impact on NCR expression on CD8 T cells in virally suppressed HIV-infected individuals. ${ }^{24}$ In cancer patients with type II diabetes mellitus, metformin before cancer treatment was associated with a decrease in the frequency of PD- $1^{+} \mathrm{CD} 8 \mathrm{~T}$ cells. ${ }^{25}$ While we observed after a brief metformin course an increase in TIGIT $^{+} \mathrm{PD}-1^{+} \mathrm{CD} 8 \mathrm{~T}$ cells, we posit that the length of metformin augmentation likely influences the trajectories of NCR expression over time. An ongoing clinical trial comparing the impact of a short course and long course of metformin on immunophenotypes among an elderly population is underway ${ }^{26}$ and the results could inform the timing of therapy for immunomodulation in both the uninfected and HIV-infected populations.

ICBs have been a powerful tool in altering the disease progression of multiple cancers, however, only a subset of individuals respond favorably to therapy. ${ }^{27}$ Furthermore, the efficacy of metformin in lowering risk for diabetes-related endpoint is roughly $30 \% .{ }^{28}$ Differences in response both for ICB and metformin therapy may have influenced the impact of combined metformin and ICB, as we have observed a variation in individuals that had improved HIV-specific CD8 $\mathrm{T}$ cell responses to ICB with metformin. This suggests that the beneficial effects of short-course metformin may be limited on a per individual basis. Thus, identifying responsive individuals is an urgent clinical prerequisite.

Metformin alters the gut microbiome by increasing mucin-degrading Akkermansia muciniphila along with several butyrate-producing bacteria, contributing to intestinal tract integrity reducing inflammation. ${ }^{29,30}$ Moreover, gut microbiome profile has been implicated in the positive outcomes of cancer individuals receiving ICB. ${ }^{31}$ In our recent studies we have observed that colonization with specific gut mucosal microbiome in HIV-infected adults on cART were related to the degree of polyfunctional anti-HIV CD8 T cell response to ICB ${ }^{32}$ Metformin has also been shown to improve the effects of ICBmediated T cell function and tumor clearance. ${ }^{14}$ It would be interesting to evaluate the pharmacodynamics of metformin and ICB interaction in relation to its influences on gut microbiome and magnitude of ICB-enhanced HIV-specific CD8 T cell responses to identify responsive individuals.

Our data show that monocyte frequencies are stable over time and baseline frequencies can potentially predict the magnitude of improved HIV-specific CD8 T cell response to PD-L1 blockade suggesting that the presence of monocyte subsets provides favorable outcomes in ICB responses. Monocytes express PD-L1, which binds to PD-1 receptor of different immune cells, including CD8 T cells, which could imply that PD-1/ PD-L1 interactions between monocytes and T cells play an important role in the efficacy of ICB. ${ }^{9}$ Furthermore, metformin has been shown to maintain cytotoxic $\mathrm{T}$ lymphocyte activity by reducing the stability and membrane localization of PD-L1 expressed in tumor cells through $\mathrm{AMPK}^{33}$ and further studies to determine the impact of metformin on PDL1 expression in HIV infection may provide further insight into alternate mechanisms of ICB efficacy.

Metformin is known to inhibit monocyte-to-macrophage differentiation, ${ }^{34}$ decrease monocyte chemoattractant protein1 (MCP-1) ${ }^{35}$ and attenuate inflammatory cytokine release in monocytes ${ }^{17}$ that could influence the crosstalk of monocyte subset and CD8 T cells in the presence of ICB. Our findings provide support for the notion that metformin may be beneficial in improving HIV-specific CD8 T cell function in the setting of chronic HIV infection.

Metformin inhibits complex 1 in the mitochondrial respiratory chain to alter cellular bioenergetics ${ }^{36}$ and engagement of PD-1 has been shown to alter T cell metabolic reprogramming of aerobic glycolysis and increased rate of fatty acid-B oxidation (FAO) upon activation. ${ }^{37}$ Blocking this pathway in our study may have potentiated the metabolic effects of metformin on CD8 T cells, improving its activation and anti-HIV effector functions. It would be interesting to investigate the glycolytic rate of activated CD8 T cells with ICB and adjunctive metformin therapy, to determine the Warburg effect of oxidative phosphorylation (OXPHOS)/ aerobic glycolysis switching. Furthermore, FAO metabolism may play an important role in the enhancement of metformin mediated ICB.

\section{Conclusion}

In summary, our data suggest that an 8-week course of adjunctive metformin in nondiabetic virally suppressed HIVinfected individuals increases central memory CD8 T cells, which have the propensity to differentiate into effector cells that target HIV. Combinatorial effect of metformin and PD-L1 blockade improved polyfunctional HIV-specific CD8 T cell responses. Furthermore, the presence of certain monocyte subsets predicts favorable outcomes in PD-L1 blockade response.

We acknowledge several potential limitations with our study that may influence our results; these include the use of an open-label single-arm study, abbreviated 8-week length of study, limited sample size, and ICB tested ex vivo. However, the impact of our trial provides valuable insight into metformin's pleiotropic effects in the setting of chronic HIV infection. Conducting a randomized double-blind placebocontrolled study over a longer duration in a larger population will be important for future studies. Furthermore, given the number of ongoing clinical trials of PD-1 blockade in HIVinfected participants, it would be intriguing to evaluate the combination of metformin and ICB in vivo in these or future trials as our study was limited in the ability to assess both interventions.

\section{Acknowledgments}

The study team would like to gratefully thank all the study participants, Hawaii Center for AIDS staff and physicians. They thank Bristol-Myers Squibb for the donation of the PD-1 and TIGIT targeted mAbs. This work was presented in part at the 26th Conference on Retroviral and Opportunistic Infections (CROI) in March 2019, Seattle, WA.

\section{Author Contributions}

G.M.C., D.M.C., A.P.S.P., M.J.C., and L.C.N. planned and carried out the experiments. G.M.C. and A.J.P.P. took the 
lead in writing the article. D.C.C., S.A.S., and C.M.S. recruited patients. G.M.C., A.J.P.P., D.C.C., S.A.S., D.M.C., A.P.S.P., M.J.C., M.M.A., M.G., and L.C.N. provided critical feedback and helped shape the research, analysis, and article.

\section{Author Disclosure Statement}

The authors have declared that no competing interests exist.

\section{Funding Information}

The project described was supported by grant number 5U54MD007601-32 from the National Institute on Minority Health and Health Disparities (NIMHD), a component of the NIH and its contents are solely the responsibility of the author and do not necessarily represent the official view of NIMHD or NIH.

\section{Supplementary Material}

Supplementary Figure S1

Supplementary Figure S2

Supplementary Figure S3

Supplementary Figure S4

\section{References}

1. Chew GM, Fujita T, Webb GM, et al:: TIGIT marks exhausted $\mathrm{T}$ cells, correlates with disease progression, and serves as a target for immune restoration in HIV and SIV infection. PLoS Pathog 2016;12:1-28.

2. Fromentin R, Bakeman W, Lawani MB, et al.: $\mathrm{CD}^{+} \mathrm{T}$ cells expressing PD-1, TIGIT and LAG-3 contribute to HIV persistence during ART. PLoS Pathog 2016;12:1-19.

3. de Armas LR, Pallikkuth S, Rinaldi S, Pahwa R, Pahwa S: Implications of immune checkpoint expression during aging in HIV-infected people on antiretroviral therapy. AIDS Res Hum Retroviruses 2019;35:1112-1122.

4. Moon EK, Langer CJ, Albelda SM: The era of checkpoint blockade in lung cancer: Taking the brakes off the immune system. Ann Am Thorac Soc 2017;14:1248-1260.

5. Guihot A, Marcelin AG, Massiani MA, et al:: Drastic decrease of the HIV reservoir in a patient treated with nivolumab for lung cancer. Ann Oncol 2018;29:517-518.

6. Wightman F, Solomon A, Kumar SS, et al:: Effect of ipilimumab on the HIV reservoir in an HIV-infected individual with metastatic melanoma. AIDS 2015;29:504-506.

7. Gay CL, Bosch RJ, Ritz J, et al.: Clinical trial of the anti-PDL1 antibody BMS-936559 in HIV-1 infected participants on suppressive antiretroviral therapy. J Infect Dis 2017;215: 1725-1733.

8. Fromentin R, DaFonseca S, Costiniuk CT, et al.: PD-1 blockade potentiates HIV latency reversal ex vivo in CD4(+) $\mathrm{T}$ cells from ART-suppressed individuals. Nat Commun 2019;10:814.

9. Krieg C, Nowicka M, Guglietta S, et al.: High-dimensional single-cell analysis predicts response to anti-PD-1 immunotherapy. Nat Med 2018;24:144-153.

10. Viollet B, Guigas B, Garcia NS, Leclerc J, Foretz M, Andreelli F: Cellular and molecular mechanisms of metformin: An overview. Clin Sci 2011;122:253-270.

11. Vial G, Detaille D, Guigas B: Role of mitochondria in the mechanism(s) of action of metformin. Front Endocrinol 2019;10:294.
12. Pearce EL, Walsh MC, Cejas PJ, et al:: Enhancing CD8 T-cell memory by modulating fatty acid metabolism. Nature 2009;460:103-107.

13. Scharping NE, Menk AV, Whetstone RD, Zeng X, Delgoffe GM: Efficacy of PD-1 blockade is potentiated by metformin-induced reduction of tumor hypoxia. Cancer Immunol Res 2017;5:9-16.

14. Afzal MZ, Mercado RR, Shirai K: Efficacy of metformin in combination with immune checkpoint inhibitors (antiPD-1/anti-CTLA-4) in metastatic malignant melanoma. J Immunother Cancer 2018;6:64.

15. Trautmann L, Janbazian L, Chomont N, et al.: Upregulation of PD-1 expression on HIV-specific $\mathrm{CD}^{+} \mathrm{T}$ cells leads to reversible immune dysfunction. Nat Med 2006;12:11981202.

16. Scharf L, Tauriainen J, Frederiksen J, et al.: HIV-induced modifications of TIGIT expression impair CD8 T cell polyfunctionality. J Immunol 2017;198(1 Suppl):78.25.

17. Zwolak A, Słabczyńska O, Semeniuk J, Daniluk J, SzusterCiesielska A: Metformin changes the relationship between blood monocyte Toll-like receptor 4 levels and nonalcoholic fatty liver disease-ex vivo studies. PLoS One 2016; 11:e0150233.

18. Guaraldi G, Orlando G, Zona S, et al.: Premature agerelated comorbidities among HIV-infected persons compared with the general population. Clin Infect Dis 2011;53: 1120-1126.

19. Klenerman P, Hill A: T cells and viral persistence: Lessons from diverse infections. Nat Immunol 2005;6:873-879.

20. Warren JA, Clutton G, Goonetilleke N: Harnessing $\mathrm{CD}^{+} \mathrm{T}$ cells under HIV antiretroviral therapy. Front Immunol 2019; 10:291.

21. Cartwright EK, Spicer L, Smith SA, et al.: CD8 ${ }^{+}$lymphocytes are required for maintaining viral suppression in SIV-infected macaques treated with short-term antiretroviral therapy. Immunity 2016;45:656-668.

22. McKinnon LR, Ball TB, Wachihi C, et al:: Epitope crossreactivity frequently differs between central and effector memory HIV-specific CD8 ${ }^{+}$T cells. J Immunol 2007;178: 3750-3756.

23. Osborn JF, Hobbs SJ, Mooster JL, et al: : Central memory $\mathrm{CD}^{+} \mathrm{T}$ cells become $\mathrm{CD} 69^{+}$tissue-residents during viral skin infection independent of CD62L-mediated lymph node surveillance. PLOS Pathog 2019;15:e1007633.

24. Shikuma CM, Chew GM, Kohorn L, et al.: Short communication: metformin reduces CD4 $\mathrm{T}$ cell exhaustion in HIV-infected adults on suppressive antiretroviral therapy. AIDS Res Hum Retroviruses 2020;36:303-305.

25. Zhang Z, Li F, Tian Y, et al:: Metformin enhances the antitumor activity of $\mathrm{CD}^{+} \mathrm{T}$ lymphocytes via the AMPKmiR-107-Eomes-PD-1 pathway. J Immunol 2020;204:25752588.

26. ClinicalTrials.gov.Identifier NCT03713801: Impact of metformin on immunity. National Library of Medicine (US), Bethesda, MD. Available at https://clinicaltrials .gov/ct2/show/NCT03713801 (2018), accessed December 19, 2019.

27. Webb ES, Liu P, Baleeiro R, Lemoine NR, Yuan M, Wang Y-H: Immune checkpoint inhibitors in cancer therapy. J Biomed Res 2018;32:317-326.

28. UK Prospective Diabetes Study (UKPDS) Group: Effect of intensive blood-glucose control with metformin on complications in overweight patients with type 2 diabetes (UKPDS 34). Lancet 1998;352:854-865. 
29. de la Cuesta-Zuluaga J, Mueller NT, Corrales-Agudelo V, et al.: Metformin is associated with higher relative abundance of mucin-degrading Akkermansia muciniphila and several short-chain fatty acid-producing microbiota in the gut. Diabetes Care 2017;40:54-62.

30. Wu H, Esteve E, Tremaroli V, et al.: Metformin alters the gut microbiome of individuals with treatment-naive type 2 diabetes, contributing to the therapeutic effects of the drug. Nat Med 2017;23:850.

31. Fessler J, Matson V, Gajewski TF: Exploring the emerging role of the microbiome in cancer immunotherapy. J Immunother Cancer 2019;7:108.

32. SahBandara IN, Chew GM, Corley MJ, et al.: Changes in gastrointestinal microbial communities influence HIVspecific $\mathrm{CD}^{+} \mathrm{T}$-cell responsiveness to immune checkpoint blockade. AIDS 2020;34:1451-1460.

33. Cha J-H, Yang W-H, Xia W, et al.: Metformin promotes antitumor immunity via endoplasmic-reticulum-associated degradation of PD-L1. Mol Cell 2018;71:606.e7-620.e7.

34. Vasamsetti SB, Karnewar S, Kanugula AK, Thatipalli AR, Kumar JM, Kotamraju S: Metformin inhibits monocyte- tomacrophage differentiation via AMPK-mediated inhibition of STAT3 activation: Potential role in atherosclerosis. Diabetes 2015;64:2028-2041.
35. Coll B, van Wijk JPHH, Parra S, et al.: Effects of rosiglitazone and metformin on postprandial paraoxonase- 1 and monocyte chemoattractant protein-1 in human immunodeficiency virus-infected patients with lipodystrophy. Eur J Pharmacol 2006;544:104-110.

36. Owen MR, Doran E, Halestrap AP: Evidence that metformin exerts its anti-diabetic effects through inhibition of complex 1 of the mitochondrial respiratory chain. Biochem J 2000;348:607-614.

37. Patsoukis N, Bardhan K, Chatterjee P, et al:: PD-1 alters T-cell metabolic reprogramming by inhibiting glycolysis and promoting lipolysis and fatty acid oxidation. Nat Commun 2015;6:6692.

Address correspondence to: Lishomwa C. Ndhlovu Division of Infection Diseases Department of Medicine

Weill Cornell Medicine, Belfer Research Building 5th Floor, BB-520, 413 East 69th Street New York, New York 10021

USA

E-mail: lndhlovu@med.cornell.edu 